\title{
General canonical variational principle and Noether theorem, their new classical and quantum physics, solution to crisis deducing all physics laws in phase space
}

\author{
C. Huang ${ }^{1,2 *}$ and Yong-Chang Huang ${ }^{3 \dagger}$ \\ ${ }^{1}$ Lawrence Berkeley National Laboratory, 1 Cyclotron Road, Berkeley CA 94720, USA \\ ${ }^{2}$ Department of Physics and Astronomy, Purdue University, \\ 525 Northwestern Avenue, W.Lafayette, IN 47907-2036, USA \\ ${ }^{3}$ Institute of Theoretical Physics, Beijing University of Technology, Beijing 100124, China
}

(Dated: October 19, 2020)

\begin{abstract}
This paper discovers that current canonical variational principle and canonical Noether theorem of (in)finite freedom systems for different physics systems have neglected doublet extreme value processes of the general extreme value functional that both is derived by variational principle and is necessarily be taken in deriving all ( quantum ) physics laws in phase space, but which have not been done for over one century since Noether's showing her distinguished theorem, which lead to the crisis deriving all (quantum) physics laws (necessary) in phase space. We discover there is the hidden logic cycle that people assume canonical equations, and then they finally deduce canonical equations by the equivalent relation in the whole processes in all current references. We correct the current key mistake concepts that when physics systems take the variational extreme values, the appearing processes of the physics systems are real physics processes, otherwise, are virtual processes in all current references. The real physics should be what after taking the physics systems' variational extreme values, the physics systems' general extremum functional needs to further take the general extremum functional's minimum absolute extremum zero, otherwise, the appearing processes of physics systems still are virtual processes. Conservation current equations and conservation currents, in phase space, of general canonical variational principle and general canonical Noether theorem are, respectively, deduced for the first time. Using the general extremum functionals' doublet extreme value processes, the hidden logic cycle and the crisis in current canonical variational principle and current canonical Noether theorem are solved. Consequently, the new mathematical pictures, classical and quantum new physics in phase space and the new mathematical and physical doublet extremum processes for (in)finite freedom systems are discovered. General canonical variational principle and general canonical Noether theorem naturally are given, which would rewrite all the different sciences in phase space, as key tools of studying and dealing with them.
\end{abstract}

Key words: canonical variational principle, canonical Noether theorem, mathematical physics, fundamental interaction, physics law, unification theory, classical and quatum new physics in phase space

PACS numbers:

\section{I.Introduction}

The systems' behaviors can be determined by the principle of least action [1]. In science, variational principle makes problems resolved via utilizing the calculus of variations, and optimizes quantities in the variational systems [2].

Using Euler-Lagrange equations and the corresponding convervation quantity deduced from variational principle can show basic physics laws [3, 4]. Variational principle is generalized to Noether theorem by Noether's finding the transformation symmetry properties of systems, and Noether showed Euler-Lagrange equations and conservation quantities related to symmetries $[5-8]$.

Different branches of science, e.g., mathematics, physics, chemistry, astronomy, even engineering and so on, have largely used variational principle and Noether theorem as key tools studying and processing the theo-

\footnotetext{
*Electronic address: c.huang0@hotmail.com

${ }^{\dagger}$ Electronic address: ychuang@bjut.edu.cn
}

ries and practical applications of the different branches [2, 3, 9-19], [20], [21, 22].

A lot of references, e.g., [23-28] have very well investigated various variational principles and their useful actual applications, and lots of good works, e.g., [29], [30], [31] have very well researched on Noether's Theorem and the useful pratical applications in different branches of physics.

There exist the needs in advance, in current variational principle and Noether theorem, to pressume existing some conditions equivalent to Euler-Lagrange equation$\mathrm{s}$ and conservation quantities, and then deriving EulerLagrange equations and conservation quantities, which are relevant to a hidden logic cycle trouble and are no exact.

This paper discover that all the studies on variational principle and Noether theorem have neglected the key investigations for the double extremum processes relevant to the general extremum functional that is derived by the least action principle and needs to be taken in deriving all the physics laws, however, the variational principle 
and Noether theorem have not do so for over one century since Noether's showing the theorem $[5,6]$, which lead to the crisis of no objectively deriving all physics laws. Utilizing the investigations for the double extremum processes relevant to the general extremum functional in this paper, the hidden logic cycle trouble and the crisis are resolved, and the new mathematical and physical double extremum processes are discovered.

Not losing the generality, all ( quantum ) physics laws in phase space always can be expressed as some equation$\mathrm{s}$, these equations always can be viewed as some cannonical equations, the canonical equations belong to phase space's Hamiltonian description coming from configuration space's Lagrangian description with Euler-Lagrange equations via Legendre transformations [33, 34], and the canonical equations always can be deduced by the canonical variational principle and/or canonical Noether theorem [5, 6], [33, 34]. Therefore, there always exists the crisis deducing all the ( quantum ) physics laws in phase space by the canonical variational principle and canonical Noether theorem. This paper plans to mainly solve the crisis. Especially, path integral quanitzation in phase space is more general than path integral quanitzation in configuration space [33, 34], and studying the canonical variational principle and canonical Noether theorem in phase space is very useful due to the key importance of quantum physics in modern physics.

This paper's arrangements: Section 2 shows unifying investigations for canonical variational principle and canonical Noether theorem of finite freedom systems; Section 3 researches on crisis of deducing physics laws in phase space and its solution to the crisis for finite freedom systems; Section 4 shows unifying investigations for canonical variational principle and canonical Noether theorem of infinite freedom systems; Section 5 investigates crisis of deducing physics laws in phase space and its solution to the crisis for infinite freedom systems; Sect. 6 shows their discussions and applications; Section 7 displays summary and conclusions.

II.Unifying investigations for canonical variational principle and canonical Noether theorem of finite freedom systems

The mathematical expressions of the least action principle in phase space are: variational of the action during $\left[t_{1}, t_{2}\right]$ about $N$ generalized coordinates $q=\left(q^{1}, q^{2}, \ldots, q^{N}\right)$ and generalized momenta $p=\left(p_{1}, p_{2}, \ldots, p_{N}\right)$ is $[18,32]$

$\Delta A=A^{\prime}-A=\int_{t_{1}^{\prime}}^{t_{2}^{\prime}} L^{\prime}\left(q^{\prime}, p^{\prime}, t^{\prime}\right) d t^{\prime}-\int_{t_{1}}^{t_{2}} L(q, p, t) d t=0$.

The general infinitesimal transformations in phase space are [32-34]

$$
\begin{gathered}
t^{\prime}=t^{\prime}(q, p, t, \alpha)=t+\Delta t=t+\varepsilon_{\sigma} \tau^{\sigma}(q, p, t, \alpha), \\
q^{i}\left(t^{\prime}\right)=q^{i}(q, p, t, \alpha)=q^{i}(t)+\Delta q^{i}=q^{i}(t)+\varepsilon_{\sigma} \xi^{i \sigma}(q, p, t, \alpha),
\end{gathered}
$$

$p_{i}^{\prime}\left(t^{\prime}\right)=p_{i}^{\prime}(q, p, t, \alpha)=p_{i}(t)+\Delta p_{i}=p_{i}(t)+\varepsilon_{\sigma} \eta_{i}^{\sigma}(q, p, t, \alpha)$,

where $\alpha=\left(\alpha_{1}, \alpha_{2}, \ldots, \alpha_{m}\right)$ are Lie group G's independent continuous variable parameters and

$$
\begin{gathered}
\tau^{\sigma}=\left.\frac{\partial t^{\prime}(q, p, t, \alpha)}{\partial \alpha_{\sigma}}\right|_{\alpha=0}, \sigma=1,2, \ldots, m . \\
\xi^{i \sigma}=\left.\frac{\partial q^{\prime}(q, p, t, \alpha)}{\partial \alpha_{\sigma}}\right|_{\alpha=0}, \sigma=1,2, \ldots, m, \\
\eta_{i}^{\sigma}=\left.\frac{\partial p_{i}^{\prime}(q, p, t, \alpha)}{\partial \alpha_{\sigma}}\right|_{\alpha=0}, \sigma=1,2, \ldots, m,
\end{gathered}
$$

Eqs.(5), (6) and (7) are the group G's infinitesimal generating functions, $\varepsilon_{\sigma}(\sigma=1,2, \ldots, \mathrm{m})$ are independent infinitesimal parameters related to $\alpha$, the curves $q(t), p(t)$ are parameterized by time, and the path in phase space takes extremum related to $\Delta A=0$.

Similar to the well known Refs. [3, 12, 18, 32, 34], one can define

$L^{p \prime}\left(q^{\prime}\left(t^{\prime}\right), p^{\prime}\left(t^{\prime}\right), t^{\prime}\right)=L^{p}\left(q^{\prime}\left(t^{\prime}\right), p^{\prime}\left(t^{\prime}\right), t^{\prime}\right)+\varepsilon_{\sigma} \frac{d \Omega^{\sigma}(q(t), p(t), t)}{d t}$

in which $\sigma=1,2, \ldots, m$.

Substituting Eq.(8) into Eq.(1), we have

$$
\begin{gathered}
\Delta A=\int_{t_{1}^{\prime}}^{t_{2}^{\prime}}\left[L^{p}\left(q^{\prime}\left(t^{\prime}\right), p^{\prime}\left(t^{\prime}\right), t^{\prime}\right)+\varepsilon_{\sigma} \frac{d \Omega^{\sigma}(q(t), p(t), t)}{d t}\right] d t^{\prime} \\
-\int_{t_{1}}^{t_{2}} L^{p}(q(t), p(t), t) d t=0
\end{gathered}
$$

where

$$
L^{p}(q(t), p(t), t)=p_{i}(t) \dot{q}^{i}(t)-H_{c}(q(t), p(t), t)
$$

Simplifying Eq.(9) and neglecting high order infinitesimal quantities, we can obtain

$$
\begin{gathered}
\Delta A=\int_{t_{1}}^{t_{2}}\left[\varepsilon_{\sigma} \frac{d \Omega^{\sigma}}{d t}+\left(\dot{q}^{i}-\frac{\partial H_{c}}{\partial p_{i}}\right) \delta p_{i}-\left(\dot{p}_{i}+\frac{\partial H_{c}}{\partial q^{i}}\right) \delta q^{i}\right. \\
\left.+\frac{d}{d t}\left(p_{i} \delta q^{i}+L^{p} \Delta t+\Omega\right)\right] d t
\end{gathered}
$$

Then Eq.(11) can be further simplified as

$$
\begin{aligned}
& \Delta A=\int_{t_{1}}^{t_{2}}\left[\left(\dot{q}^{i}-\frac{\partial H_{c}}{\partial p_{i}}\right) \delta p_{i}-\left(\dot{p}_{i}+\frac{\partial H_{c}}{\partial q^{i}}\right) \delta q^{i}\right. \\
& \left.\frac{d}{d t}\left(p_{i} \delta q^{i}+L^{p} \Delta t+\Omega\right)\right] d t=0
\end{aligned}
$$


in which $\Omega=\varepsilon_{\sigma} \Omega^{\sigma}$.

No lossing generality, there are still three different situations:

Situation (a): When presuming

$$
\Delta A=\int_{t_{1}}^{t_{2}} \frac{d}{d t}\left[p_{i} \delta q^{i}+L^{p} \Delta t+\Omega\right] d t,
$$

utilzing Eq.(12) and Eq.(13), we achieve

$$
\dot{q}^{i}=\frac{\partial H_{c}}{\partial p_{i}}, \dot{p}_{i}=-\frac{\partial H_{c}}{\partial q^{i}},
$$

in which $i=1,2, \ldots, N$, due to the linear independent properties of $\delta q^{i}$ and $\delta p_{i}(\mathrm{i}=1,2, \ldots, \mathrm{N})$.

Utilizing Eq.(13), one can derive conservation quantity

$$
p_{i} \delta q^{i}+L^{p} \Delta t+\Omega=\text { const. }
$$

Using

$$
\Delta q^{i}=\delta q^{i}(t)+\dot{q}^{i}(t) \Delta t, \Delta p_{i}=\delta p_{i}(t)+\dot{p}_{i}(t) \Delta t,
$$

we can rewrite Eq.(15) as

$$
p_{i}\left(\Delta q^{i}-\dot{q}^{i}(t) \Delta t\right)+L^{p} \Delta t+\Omega=\text { const } .
$$

Eq.(17) is the conservation quantity of the systems from canonical variational principle.

Substituting Eqs.(2) \& (3) into Eq.(17), we deirve m conservation quantities of the systems

$$
p_{i}\left(\xi^{i \sigma}-\dot{q}^{i}(t) \tau^{\sigma}\right)+L^{p} \tau^{\sigma}+\Omega^{\sigma}=\text { const }^{\sigma} .
$$

in which $\varepsilon_{\sigma}(\sigma=1,2, \ldots, m)$ belonging independent infinitesimal parameters have been used. That is, Eq.(18) is Noetther theorem's conservation charges [18, 34].

One can watch that variational principle \& Noether theorem both give the same canonical equations 14), but their convervation quantities are Eq.(17) and Eq.(18) respectively, i.e., are very different.

Situation (b): When presuming that there is Eq.(14), and then substituting Eq.(14) into Eq.(12), we have Eq.(13). There are, in the following, the very similar discussions underneath Eq.(14) in situation (a).

III.Crisis deducing physics laws in phase space and solution to the crisis for finite freedom systems

Situation (c): Utilzing Eq.(12) and merging like terms, one exactly has a general functional

$$
\begin{aligned}
& \int_{t_{1}}^{t_{2}}\left[\left(\dot{q}^{i}-\frac{\partial H_{c}}{\partial p_{i}}\right) \delta p_{i}-\left(\dot{p}_{i}+\frac{\partial H_{c}}{\partial q^{i}}\right) \delta q^{i}\right] d t \\
=- & \int_{t_{1}}^{t_{2}} \frac{d}{d t}\left(p_{i} \delta q^{i}+L^{p} \Delta t+\Omega\right) d t
\end{aligned}
$$

Eq.(19) is deduced by taking extremum of the general Lagrangian systems, when the systems don't have Eq.(13) or Eq.(14), the systems then cannot give canonical equations and the corresponding conservation quantity. That is, this situation cannot show real physics laws, which just is the reason that both canonical variational principle and canonical Noether theorem have neglected the situation (c) $[18,34]$.

Utilzing Eq.(19) deduced by the variational extremum, we are able to define exactly a general extremum functional

$$
\begin{aligned}
& F=\int_{t_{1}}^{t_{2}}\left[\left(\dot{q}^{i}-\frac{\partial H_{c}}{\partial p_{i}}\right) \delta p_{i}-\left(\dot{p}_{i}+\frac{\partial H_{c}}{\partial q^{i}}\right) \delta q^{i}\right] d t \\
& =-\int_{t_{1}}^{t_{2}} \frac{d}{d t}\left(p_{i} \delta q^{i}+L^{p} \Delta t+\Omega\right) d t
\end{aligned}
$$

The general new functional $F$ between the functional $F_{c a}$ for deducing canonical equations having merged liketerms relevant to canonical equations and the functional $F_{c o}$ for deducing the general conservation quantities having merged like-terms relevant to the general conservation quantities is derived by satisfying variational principle, namely, $F=F_{c a}=-F_{c o}$, namely, $F_{c a}+F_{c o}=0$, which shows just the variational extremum, but these cannot still show real physics ( see the investigations below ), these are the very key classical and quantum new physics processes for the general physics systems.

When the minimum absolute value of the general extremum function $F$ is taken as zero, because the minimum absolute value of any function is zero, namely, a general extremum, we, then, have

$$
\begin{gathered}
\int_{t_{1}}^{t_{2}}\left[\left(\dot{q}^{i}-\frac{\partial H_{c}}{\partial p_{i}}\right) \delta p_{i}-\left(\dot{p}_{i}+\frac{\partial H_{c}}{\partial q^{i}}\right) \delta q^{i}\right] d t=0 \\
=-\int_{t_{1}}^{t_{2}} \frac{d}{d t}\left(p_{i} \delta q^{i}+L^{p} \Delta t+\Omega\right) d t=0 .
\end{gathered}
$$

Eq.(21)'s the first line is just equivalent to situation (b), and Eq.(21)'s the second is equivalent to situation (a), which can show physics laws. That is, the general extremum functional $F$ chooses a minimum absolute zero value, the physics laws are able to be deduced. Therefore, we discover that the extreme functional $F$ 's extremum leads to that the physics laws can be derived.

The systems, thus, first choose the extremum of the Lagrangian by Eq.(1), need in advance as usual to presume existing situation (a) or (b), which are equivalent to canonical equations and conservation quantities, and then deriving canonical equations and conservation quantities, which are relevant to a hidden logic cycle trouble and are not natural and exact.

There actually naturally is the general extremum functional $F$ such that one can take the general extremum 
functional $F$ 's the absolute extreme value zero, and then situation (a) or (b) is able to be naturally derived, e.g., refer the discussions below Eq.(21). The natural deductions show the systems' intrinsical properties, that is, the objective double extremum procceses in mathematics and physics. Otherwise the systems are not able to obtain real physical laws. These results are not only supplementary developments of the current canonical variational principle and current canonical Noether theorem but also classsical and quantum new physics corresponding to classical and quantum canonical physics systems, because this Lagrangian is a general Lagrangian.

Up to now, we discover that all the studies on canonical variational principle and canonical Noether theorem about different physics systems have neglected the key investigations for the double extremum processes relevant to the general extremum functional $F$ which is derived by the least action principle and needs to be largely taken in deriving the physics laws in phase space, however, the current canonical variational principle and current canonical Noether theorem have missed the general extremum function $F$ \& F's minimum extremum, which lead to the hidden logic cycle disaster and the crisis of no objectively deriving all physics laws in phase space. Using the investigations about the double extremum processes relevant to the general extremum functional $F$ in phase space, the hidden logic cycle disaster and the crisis are solved, and the new double extremum processes and the new pictures in mathematics and physics are naturally discovered. Consequently, the canonical canonical variantional principle and the canonical canonical Noether theorem of finite freedom systems are shown, which resolve the hidden logic cycle disaster and the crisis.

IV.Unifying investigations of canonical variational principle and canonical Noether theorem for infinite freedom systems

Considering the exact mathematical expressions for the least action principle in a general situation are: variational of the action for $N$ field components $X=$ $\left(X^{1}, X^{2}, \ldots, X^{N}\right)$, e.g., general field variables $X(x)=$ $\left\{\Psi(x), \varphi(x), \omega_{\mu}(x), g_{\mu \nu}(x), \ldots,\right\}$, and momenta $\Pi=$ $\left(\Pi^{1}, \Pi^{2}, \ldots, \Pi^{N}\right)$ is $[33,34]$

$$
\begin{aligned}
\Delta A & =A^{\prime}-A=\int_{x_{1}^{\prime}}^{x_{2}^{\prime}} \mathcal{L}^{p^{\prime}}\left(X^{\prime}\left(x^{\prime}\right), \Pi^{\prime}\left(x^{\prime}\right), x^{\prime}\right) d x^{4} \\
& -\int_{x_{1}}^{x_{2}} \mathcal{L}^{p}\left((X(x), \Pi(x), x) d x^{4}=0 .\right.
\end{aligned}
$$

where general infinitesimal transformations are [33, 34]

$$
\begin{gathered}
x^{\prime \mu}=x^{\mu}+\Delta x^{\mu}=x^{\mu}+\varepsilon_{\sigma}(x) \tau^{\mu \sigma}(x, X(x), \Pi(x)), \\
X^{\prime \alpha}\left(x^{\prime}\right)=X^{a}(x)+\varepsilon_{\sigma}(x) \xi^{a \sigma}(x, X(x), \Pi(x))
\end{gathered}
$$

$$
\Pi^{\prime \alpha}\left(x^{\prime}\right)=\Pi^{a}(x)+\varepsilon_{\sigma}(x) \eta^{a \sigma}(x, X(x), \Pi(x))
$$

in which $X^{\prime \alpha}\left(x^{\prime}\right)=X^{\alpha}(x)+\Delta X^{\alpha}(x), \Pi^{\prime \alpha}\left(x^{\prime}\right)=\Pi^{\alpha}(x)+$ $\Delta \Pi^{\alpha}(x), \omega=\left(\omega_{1}, \omega_{2}, \ldots, \omega_{m}\right)$ are Lie group $G^{\prime}$ 's independent continuous variable parameters and

$$
\begin{gathered}
\tau^{\mu \sigma}=\left.\frac{\partial x^{\mu}(x, X(x), \Pi(x), \omega)}{\partial \omega_{\sigma}}\right|_{\omega_{\sigma}=0}, \\
\xi^{a \sigma}=\left.\frac{\partial X^{\alpha}(x, X(x), \Pi(x), \omega)}{\partial \omega_{\sigma}}\right|_{\omega_{\sigma}=0}, \\
\eta^{a \sigma}=\left.\frac{\partial \Pi^{\alpha}(x, X(x), \Pi(x), \omega)}{\partial \omega_{\sigma}}\right|_{\omega_{\sigma}=0},
\end{gathered}
$$

in which $\tau^{\mu \sigma}, \xi^{a \sigma}$ and $\eta^{a \sigma}(\sigma=1,2, \ldots, m)$ are infinitesimal transformation functions $[33,34]$.

Eqs.(26), (27) and (28) are the group G's infinitesimal generating functions, $\varepsilon_{\sigma}$ are independent infinitesimal parameters related to $\omega$.

No loss of generality, we can define

$$
\begin{gathered}
\mathcal{L}^{p \prime}\left(X^{\prime}\left(x^{\prime}\right), \Pi^{\prime}\left(x^{\prime}\right), x^{\prime}\right)=\mathcal{L}^{p}\left(X^{\prime}\left(x^{\prime}\right), \Pi^{\prime}\left(x^{\prime}\right), x^{\prime}\right) \\
+\varepsilon_{\sigma} \partial_{\mu} \Omega^{\mu \sigma}(X(x), \Pi(x), x),
\end{gathered}
$$

$$
\mathcal{L}^{p}(X(x), \Pi(x), x)=\Pi_{a}(x) \dot{X}^{a}(x)-\mathcal{H}_{c}(X(x), \Pi(x), x)
$$

where $H_{c}=\int_{M_{3}} \mathcal{H}_{c} d x^{3}$. Substituting Eq.(29) into Eq.(22), we have

$$
\begin{aligned}
\Delta A & =\int_{x_{1}^{\prime}}^{x_{2}^{\prime}}\left[\mathcal{L}^{p}\left(X^{\prime}\left(x^{\prime}\right), \Pi^{\prime}\left(x^{\prime}\right), x^{\prime}\right)+\varepsilon_{\sigma} \partial_{\mu} \Omega^{\mu \sigma}(X(x), \Pi(x), x)\right. \\
& \left.-\mathcal{L}^{p}\left(X\left(x^{\prime}\right), \Pi\left(x^{\prime}\right), x^{\prime}\right)+\mathcal{L}^{p}\left(X\left(x^{\prime}\right), \Pi\left(x^{\prime}\right), x^{\prime}\right)\right] d x^{\prime 4}-
\end{aligned}
$$$$
\int_{x_{1}}^{x_{2}} \mathcal{L}^{p}(X(x), \Pi(x), x) d x^{4}=\int_{x_{1}^{\prime}}^{x_{2}^{\prime}}\left[\delta \mathcal{L}^{p}\left(X^{\prime}\left(x^{\prime}\right), \Pi^{\prime}\left(x^{\prime}\right), x^{\prime}\right)\right.
$$$$
+\varepsilon_{\sigma} \partial_{\mu} \Omega^{\mu \sigma}(X(x), \Pi(x), x)+\mathcal{L}^{p}(X(x), \Pi(x), x)+
$$$$
\left.\frac{D \mathcal{L}^{p}}{D x^{\mu}} \Delta x^{\mu}\right]\left(1+\frac{\partial \Delta x^{\mu}}{\partial x^{\mu}}\right) d x^{4}-\int_{x_{1}}^{x_{2}} \mathcal{L}^{p}(X(x), \Pi(x), x) d x^{4}
$$$$
=\int_{x_{1}}^{x_{2}}\left[\left(\frac{\partial\left(\Pi_{b} \dot{X}^{b}-\mathcal{H}_{c}\right)}{\partial X^{a}} \delta X^{a}+\frac{\partial\left(\Pi_{b} \dot{X}^{b}-\mathcal{H}_{c}\right)}{\partial \Pi_{a}} \delta \Pi_{a}\right.\right.
$$$$
\left.+\varepsilon_{\sigma} \partial_{\mu} \Omega^{\mu \sigma}(X(x), \Pi(x), x)+\frac{D \mathcal{L}^{p}}{D x^{\mu}} \Delta x^{\mu}+\mathcal{L}^{p} \frac{\partial \Delta x^{\mu}}{\partial x^{\mu}}\right] d x^{4}
$$ 
where $D \mathcal{L}^{p} / D x^{\mu}$ is whole derivative of the canonical Lagrangian. Making Eq.(31) into order and missing high order infinitesimal quantities, we achieve

$$
\begin{aligned}
\Delta A= & \int_{x_{1}}^{x_{2}}\left[\left(-\dot{\Pi}^{b}-\frac{\partial \mathcal{H}_{c}}{\partial X^{a}}\right) \delta X^{a}+\left(\dot{X}^{a}-\frac{\partial \mathcal{H}_{c}}{\partial \Pi_{a}}\right) \delta \Pi_{a}\right. \\
& \left.+D\left(\Pi_{a} \delta X^{a}\right)+\frac{\partial}{\partial x^{\mu}}\left(\mathcal{L}^{p} \Delta x^{\mu}+\varepsilon_{\sigma} \Omega^{\mu \sigma}\right)\right] d x^{4}
\end{aligned}
$$

Eq.(32) can be further simplified as

$$
\begin{aligned}
\Delta A= & 0=\int_{x_{1}}^{x_{2}}\left[\left(\dot{X}^{a}-\frac{\partial \mathcal{H}_{c}}{\partial \Pi^{a}}\right) \delta \Pi_{a}-\left(\dot{\Pi}^{b}+\frac{\partial \mathcal{H}_{c}}{\partial X^{a}}\right) \delta X^{a}\right. \\
& \left.+D\left(\Pi_{a} \delta X^{a}\right)+\frac{\partial}{\partial x^{\mu}}\left(\mathcal{L}^{p} \Delta x^{\mu}+\Omega^{\mu}\right)\right] d x^{4}
\end{aligned}
$$

where $\Omega^{\mu}=\varepsilon_{\sigma} \Omega^{\mu \sigma}$ is an infinitesimal quantity of one order. Eq.(33) cannot directly show canonical equation$\mathrm{s}$, because there exist some additional degrees of choice freedom.

There are still three situations for Eq.(33):

Situation (A): When presuming

$$
\int_{x_{1}}^{x_{2}}\left[D\left(\Pi_{a} \delta X^{a}\right)+\frac{\partial}{\partial x^{\mu}}\left(\mathcal{L}^{p} \Delta x^{\mu}+\Omega^{\mu}\right)\right] d x^{4}=0,
$$

utilizing Eq.(34), we have

$$
\dot{X}^{a}=\frac{\partial \mathcal{H}_{c}}{\partial \Pi_{a}}, \dot{\Pi}_{a}=-\frac{\partial \mathcal{H}_{c}}{\partial X^{a}}
$$

because $\delta X^{a}$ and $\delta \Pi_{a}(a=1,2, \ldots, N)$ are, each other, independent. Eq.(35) just are the usual canonical equations.

Utilizing Eq.(34) and Gauss theorem equating zero on boundary surfaces, we derive a general equation

$$
\left.\int_{x_{1}}^{x_{2}} \partial_{0}\left[\Pi_{a}\left(\delta X^{a}+\dot{X}^{a} \Delta x^{0}\right)-\mathcal{H}_{c} \Delta x^{0}+\Omega^{0}\right)\right] d x^{4}=0
$$

that is

$$
\begin{gathered}
\left.\int_{x_{1}}^{x_{2}}\left[\Pi_{a}\left(\Delta X^{a}-X,{ }_{\nu}^{a} \Delta x^{\nu}+\dot{X}^{a} \Delta x^{0}\right)-\mathcal{H}_{c} \Delta x^{0}+\Omega^{0}\right)\right] d x^{3}= \\
\int_{x_{1}}^{x_{2}}\left[\Pi_{a}\left(\Delta X^{a}-X_{,}^{a} \Delta x^{i}\right)-\mathcal{H}_{c} \Delta x^{0}+\Omega^{0}\right] d x^{3}=\text { const. }
\end{gathered}
$$

Eq. (37) is just the consevation quantity of canonical variational principle.

Utilizing Eq. (23) and Eq.(24), we can rewrite Eq. (37) as

$$
\int_{x_{1}}^{x_{2}}\left[\Pi_{a}\left(\xi^{a \sigma}-X,_{i}^{a} \tau^{i \sigma}\right)-\mathcal{H}_{c} \tau^{0 \sigma}+\Omega^{0 \sigma}\right] d x^{3}=\text { const }^{\sigma}
$$

Eq. (38) is just the consevation quantities of canonical Noether theorem.

On the other hand, using Eqs. (34), we can have

$$
\begin{aligned}
& \left.\int_{x_{1}}^{x_{2}} \partial_{\mu}\left[\Pi_{a} \delta X^{a} \delta^{\mu 0}+\left(\Pi_{a} \dot{X}^{a}-\mathcal{H}_{c}\right) \Delta x^{\mu}+\Omega^{\mu}\right)\right] d x^{4}=0= \\
& \left.\int_{x_{1}}^{x_{2}} \partial_{\mu}\left[\Pi_{a}\left(\delta X^{a} \delta^{\mu 0}+\dot{X}^{a} \Delta x^{\mu}\right)-\mathcal{H}_{c} \Delta x^{\mu}+\Omega^{\mu}\right)\right] d x^{4}=0
\end{aligned}
$$

utilizing Eqs. (39), we achieve the new convervation current of improved canonical variational principle

$$
J^{\mu}=\Pi_{a}\left(\left(\Delta X^{a}-X_{, \nu}^{a} \Delta x^{\nu}\right) \delta^{\mu 0}+\dot{X}^{a} \Delta x^{\mu}\right)-\mathcal{H}_{c} \Delta x^{\mu}+\Omega^{\mu}
$$

Using $\delta X^{a}=\Delta X^{a}-X_{, \nu}^{a} \Delta x^{\nu}$, Eq.(23) and Eq.(24), we achieve $\mathrm{m}$ continuos equations and their conservative currents

$$
\partial_{\mu} J^{\mu \sigma}=\partial_{\mu}\left[\Pi_{a}\left(\left(\Delta X^{a}-X_{, \nu}^{a} \Delta x^{\nu}\right) \delta^{\mu 0}+\dot{X}^{a} \Delta x^{\mu}\right)\right.
$$

$$
\left.-\mathcal{H}_{c} \Delta x^{\mu}+\Omega^{\mu}\right]=0
$$

$\partial_{\mu} J^{\mu \sigma}=\partial_{\mu}\left[\Pi_{a}\left(\left(\xi^{a \sigma}-X_{, \nu}^{a} \tau^{\nu \sigma}\right) \delta^{\mu 0}+\dot{X}^{a} \tau^{\mu \sigma}\right)-\mathcal{H}_{c} \tau^{\mu \sigma}+\Omega^{\mu \sigma}\right]=0$

$J^{\mu \sigma}=\Pi_{a}\left(\left(\xi^{a \sigma}-X_{, \nu}^{a} \tau^{\nu \sigma}\right) \delta^{\mu 0}+\dot{X}^{a} \tau^{\mu \sigma}\right)-\mathcal{H}_{c} \tau^{\mu \sigma}+\Omega^{\mu \sigma}$

in which we have utilized that $\varepsilon_{\sigma}(\sigma=1,2, \ldots, m)$ are independent infinitesimal parameters. That is, Eq.(42) is the new canonical Noetther theorem's conservation currents.

Utilizing Eq.(40) and Eq.(42) and Gauss theorem $\int_{M^{3}} \partial_{0} J^{0} d V=-\int_{M^{3}} \partial_{i} J^{i} d V=-\int_{M^{2}} J^{i} d S_{i} \rightarrow 0,\left(S_{i} \rightarrow\right.$ $\infty, J^{i} \rightarrow 0, M^{2}$ is the $M^{3}$ 's closed surface), we achieve conservation charges of improved cannonical variational principle and improved cannonical Noether theorem, respectively

$$
Q_{v p}=\int_{M^{3}} J^{0} d x^{3}, Q_{N t}^{\sigma}=\int_{M^{3}} J^{0 \sigma} d x^{3}, \sigma=1,2, \ldots, m .
$$

They are just Eq.(37) and Eq.(38), which just display that our investigations are consistent with the above two different reasonings. 
One can watch that improved canonical variational principle and improved canonical Noether theorem both give the same canonical equations (35), but they give that the convervation currents are Eq.(40) and Eq.(42) respectively, i.e., are very different.

Situation (B): When presuming that there exist Eq.(35), then substituting Eq.(35) into Eq.(33), we have Eq.(34). There are the very similar discussions in the following below Eq.(35) in situation (A).

V.Crisis of deducing physics laws in phase space and its solution to the crisis for infinite freedom systems

Situation (C): Utilizing Eq.(33), we can generally obtain

$$
\begin{aligned}
& \int_{x_{1}}^{x_{2}}\left[\left(\dot{X}^{a}-\frac{\partial \mathcal{H}_{c}}{\partial \Pi_{a}}\right) \delta \Pi_{a}-\left(\dot{\Pi}^{b}+\frac{\partial \mathcal{H}_{c}}{\partial X^{a}}\right) \delta X^{a}\right] d^{4} x= \\
& -\int_{x_{1}}^{x_{2}}\left[D\left(\Pi_{a} \delta X^{a}\right)+\frac{\partial}{\partial x^{\mu}}\left(\mathcal{L}^{p} \Delta x^{\mu}+\Omega^{\mu}\right)\right] d^{4} x
\end{aligned}
$$

Eq.(44) is deduced by taking extremum of the general Lagrangian systems, when the systems don't have Eq.(34) or Eq.(34), the systems then cannot give canonical equations and the corresponding conservation quantity. That is, this situation cannot show real physics laws, which just is the reason that both canonical variational principle and canonical Noether theorem have neglected the situation $(\mathrm{C})$.

Utilzing Eq.(44) deduced by the variational extremum, we are able to define exactly a general extremum functional

$$
\begin{aligned}
G & =\int_{x_{1}}^{x_{2}}\left[\left(\dot{X}^{a}-\frac{\partial \mathcal{H}_{c}}{\partial \Pi_{a}}\right) \delta \Pi_{a}-\left(\dot{\Pi}_{a}+\frac{\partial \mathcal{H}_{c}}{\partial X^{a}}\right) \delta X^{a}\right] d^{4} x= \\
& -\int_{x_{1}}^{x_{2}}\left[D\left(\Pi_{a} \delta X^{a}\right)+\frac{\partial}{\partial x^{\mu}}\left(\mathcal{L}^{p} \Delta x^{\mu}+\Omega^{\mu}\right)\right] d^{4} x
\end{aligned}
$$

The general new equal functional $G$ between the functional $G_{c a}$ for deducing canonical equations having merged like-terms relevant to canonical equations and the functional $G_{c o}$ for deducing the general conservation quantities having merged like-terms relevant to the general conservation quantities is derived by satisfying variational principle, namely, $G=G_{c a}=-G_{c o}$, namely, $G_{c a}+G_{c o}=0$, which shows just the variational extremum, but these cannot still show real physics ( see the investigations below ), these are the very key classical and quantum new physics processes for the general physics systems.

When the minimum absolute value of the general extremum function $G$ is taken as zero, because the minimum absolute value of any function is zero, namely, a general extremum, we, then, have

$$
\begin{aligned}
& \int_{x_{1}}^{x_{2}}\left[\left(\dot{X}^{a}-\frac{\partial \mathcal{H}_{c}}{\partial \Pi_{a}}\right) \delta \Pi_{a}-\left(\dot{\Pi}_{a}+\frac{\partial \mathcal{H}_{c}}{\partial X^{a}}\right) \delta X^{a}\right] d^{4} x=0= \\
& -\int_{x_{1}}^{x_{2}}\left[D\left(\Pi_{a} \delta X^{a}\right)+\frac{\partial}{\partial x^{\mu}}\left(\mathcal{L}^{p} \Delta x^{\mu}+\Omega^{\mu}\right)\right] d^{4} x=0 .
\end{aligned}
$$

Eq.(46)'s the first line is just equivalent to situation (B), and Eq.(46)'s the second is equivalent to situation (A), which can show physics laws. That is, the general extremum functional $G$ chooses a minimum absolute zero value, the physics laws are able to be deduced. Therefore, we discover that the extreme functional $G$ 's extremum leads to that the physics laws can be derived.

The systems, thus, first choose the extremum of the Lagrangian by Eq.(22), and we, then, naturally derive Eq.(33), need in advance as usual to presume existing situation $(\mathrm{A})$ or $(\mathrm{B})$, which are equivalent to canonical equations and conservation quantities, and then deriving canonical equations and conservation quantities, which are relevant to a hidden logic cycle trouble and are not natural and exact.

There actually naturally is the general extremum functional $G$ such that one can take the general extremum functional $G$ 's the absolute extreme value zero, and then situation (A) or (B) is able to be naturally derived, e.g., refer the discussions below Eq.(46). The natural deductions shows the systems' intrinsical properties, that is, the objective double extremum procceses in mathematics and physics. Otherwise the systems are not able to obtain real physical laws. These results are not only supplementary developments of the current canonical variational principle and current canonical Noether theorem, but also classsical and quantum new physics corresponding to classical and quantum canonical physics systems, because this Lagrangian is a general Lagrangian.

Up to now, we discover that all the studies on canonical variational principle and canonical Noether theorem about different physics systems have neglected the key investigations for the double extremum processes relevant to the general extremum functional $G$ which is derived by the least action principle and needs to be largely taken in deriving the physics laws in phase space, however, the current canonical variational principle and current canonical Noether theorem for imfinite freedom systems have missed the general extremum function $G \& G$ 's minimum extremum, which lead to the hidden logic cycle disaster and the crisis of no objectively deriving all physics laws in phase space. Using the investigations about the double extremum processes relevant to the general extremum functional $G$ in phase space, the hidden logic cycle disaster and the crisis are solved, and the new double extremum processes and the new pictures in mathematics and physics are naturally discovered. Consequently, the general canonical variantional principle and the general canonical Noether theorem of infinite freedom systems are shown, which resolve the hidden logic cycle disaster and the crisis. 


\section{Discussions and applications}

For finite freedom systems, when replacing $t, q^{i}(t), p_{i}(t)$ in eq.(1), respectively, with $x, X^{a}(x), \Pi^{a}(x)$ for infinite freedom systems, e.g., general field variables $X^{a}(x)=$ $\left\{\psi(x), \phi(x), \omega_{\alpha}(x), g_{\alpha \beta}(x)\right\}$, people can derive canonical equations Eq.(35) and the relevant conservation currents Eq.(40) and Eq.(42) of the improved canonical variational principle and the improved canonical Noether theorem, respectively, for infinite freedom systems in phase space, which have the extensive uses in the different branches of modern science, for examples, in different branches of mathematics, physics, chemistry, even engineering and so on.

For the improved variational principle and improved Noether theorem for (in)finite freedom systems in configuration space has been given in works due to the length constraint of this paper [35].

Utilizing Eq.(20) deduced by the variational extremum, people can have a general extremum functional for finite freedom systems

$$
\begin{gathered}
f=\left(\dot{q}^{i}-\frac{\partial H_{c}}{\partial p_{i}}\right) \delta p_{i}-\left(\dot{p}_{i}+\frac{\partial H_{c}}{\partial q^{i}}\right) \delta q^{i} \\
=-\frac{d}{d t}\left(p_{i} \delta q^{i}+L^{p} \Delta t+\Omega\right)
\end{gathered}
$$

in which $f$ is able to take arbitrary functional value and $F=\int_{t_{1}}^{t_{2}} f d t$.

When the general extremum functional $f$ 's minimum absolute value is taken to zero, i.e., taking the general extremum functional $f$ 's the minimum absolute extremum zero, namely, the doublet extreme value process, then using Eq.(47) people can directly derive canonical equation$\mathrm{s}$ due to the independent properties of $\delta q_{i}$ and $\delta p_{i}$ each other and the general conservation quantity due to having chosen Eq.(47)'s second line into zero. Consequently, people can discover that the processes of no taking the minimum absolute extreme value of the general extremum functional $f$ statisfying the canonical variational extremum principle are still virtual processes, because this stituation cannot deduce real canonical equations and the correspoonding conservation quantity. Namely, when taking the double extremum process of the general extremum functional $f$, the processes of the systems are real physics processes and can give the canonical eqautions and the corresponding conservation quantity.

Utilizing Eq.(45) deduced by the variational extremum, for infinite freedom systems, we derive a general extremum functional

$$
\begin{aligned}
g & =\left(\dot{X}^{a}-\frac{\partial \mathcal{H}_{c}}{\partial \Pi^{a}}\right) \delta \Pi_{a}-\left(\dot{\Pi}^{b}+\frac{\partial \mathcal{H}_{c}}{\partial X^{a}}\right) \delta X^{a} \\
& =-\left[D\left(\Pi_{a} \delta X^{a}\right)+\frac{\partial}{\partial x^{\mu}}\left(\mathcal{L}^{p} \Delta x^{\mu}+\Omega^{\mu}\right)\right]
\end{aligned}
$$

in which $g$ can take arbitrary functional value and $G=$ $\int_{M^{4}} g d^{4} x$.

When the general extremum functional $g$ 's minimum absolute extremum is taken into zero, i.e., taking the general extremum functional $g$ 's the minimum absolute extremum, namely, the general extremum functional $g$ 's extreme value, i.e., the double extreme value processes, using Eq.(48), we can directly derive canonical equations due to the independent properties of $\delta X^{a}$ and $\delta \Pi_{a}$ each other and the general conservation current in phase space due to having taken Eq.(48)'s second line into zero.

Therefore, we discover that the processes no taking the minimum absolute extremum zero of the general extremum functional $F(G)$ statisfying the variational extremum principle still are the virtual processes, because all current refererenes, e.g., refs.[5, 6],[3, 15-19], think of situations (c) and (C) satisfying the variational extreme value cannot deduce cannonical equations and their correspoonding conservation quantities. Anyway, for taking the processes of the general extremum functional $F$ 's ( $G$ 's ) minimam absolute extremum zero, the physics systems' processes are just real physics processes and can give canonical eqautions and their corresponding conservation quantities. Especially, situations (a) and (b) $((\mathrm{A})$ and $(\mathrm{B}))$ are the two special taken value situations and are included in case $(c)((\mathrm{C}))$ as special situation$\mathrm{s}$, and there exists the hidden logic cycle between situation (a) (assuming to exist Eq.(13) of deducing conservation quantity, then putting Eq.(13) into Eq.(12), people can derive canonical Eq.(14) ) and situation (b) ( assuming to exist canonical Eq.(14), then putting Eq.(14) into Eq.(12), people can derive Eq.(13) of deducing conservation quantity ), namely, situations (a) and (b) are, each other, equivalent, which means that people assume canonical equations in situation (b), and then they finally derive canonical equations in situation (a) by the equivalent relation between situations (a) and (b) in the whole processes, which is just the hidden logic cycle, so does Eq.(13) of deducing conservation quantity (similar for situations (A) and (B)). Consequently, the current investigations about situations $(\mathrm{a}-\mathrm{c})((\mathrm{A}-\mathrm{C}))$ in all current references, e.g., refs.[5, 6],[3, 15-19], are not the exact general investigations.

Therefore, we correct the current key mistakes that when physics systems take the variational extreme values, the appearing processes of the physics systems are real physics processes, otherwise, are virtual processes in all current articles, reviews and (text)books, e.g., $[5,6],[3,15-19]$. The real physics should be what after taking the variational extreme values of physics systems, the physics systems' general extremum functional $F(G)$ needs to further take the general extremum functional $F$ 's $(G$ 's ) minimum absolute extremum zero, otherwise, the appearing processes of physics systems still are virtual processes because the virtual process situations cannot deduce canonical equations and their corresponding conservation quantities.

In this paper, all the studies on functionals $F$ and $G$ 
give the relevant integral expressions, utilizing functional $f$ and $g$ people can show the relevant differantial expressions, the two expressions are whole equivalent, this paper, thus, doesn't repeat more here.

\section{Summary and conclutions}

One can derive canonical equations and corresponding conservation quantities via using canonical variational principle and canonical Noether theorem of the systems with canonical Lagrangian and symmetry of finite (infinite) freedoms. But this paper discovers that the systems have generally intrinsical freedoms of extra choices. If no to presume existing Eq.(13) or Eq.(14) (Eq.(34) or Eq.(35)), the canonical Lagrange systems, then, cannot show true physical laws. Eq.(13) and Eq.(14) (Eq.(34) and (35)) are actually equivalent to canonical equations and conservation quantities, and then deriving canonical equations and conservation quantities, which are relevant to a hidden logic cycle and are no both natural and exact.

We discover that the processes no taking the minimum absolute extremum zero of the general extremum functional $F(G)$ statisfying the variational extremum principle still are the virtual processes, because all curren$t$ refererenes think of situations (c) and (C) satisfying the variational extreme value cannot deduce cannonical equations and their correspoonding conservation quantity. We discover that for further taking the processes of the general extremum functional $F$ 's ( $G$ 's ) minimam absolute extremum zero, the physics systems' processes are just real physics processes and can give canonical eqautions and their corresponding conservation quantities, which are the new key physics.

Especially, situations (a) and (b) ((A) and (B)) are included in case $(c)((C))$ as special situations, and there exists the hidden logic cycle between situation (a) ( assuming to exist Eq.(13) ( Eq.(34) ) of deducing conservation quantity, then putting Eq.(13) ( Eq.(34) ) into Eq.(12) ( Eq.(33)), people can derive canonical Eq.(14) ( Eq.(35) ) and situation (b) ( assuming to exist canonical Eq.(14) ( Eq.(35)), then putting Eq.(14) ( Eq.(35) ) into Eq.(12) ( Eq.(33)), people can derive Eq.(13) ( Eq.(34) ) of deducing conservation quantity ), namely, situations (a) and (b) ((A) and (B)) are, each other, equivalent, which means that people assume canonical equations in situation (b) (B), and then they finally derive canonical equations in situation (a) (A) by the equivalent relation between situations (a) and (b) ( (A) and (B) ) in the whole processes, which is just the hidden logic cycle, so does Eq.(13) ( Eq.(34) ) of deducing conservation quantity. Consequently, the current investigations about situations $(\mathrm{a}-\mathrm{c})((\mathrm{A}-\mathrm{C}))$ in all current references are no the exact general investigations.

Therefore, we correct the current key mistake concepts that when physics systems take the variational extreme values, the appearing processes of the physics systems are real physics processes, otherwise, are virtual processes in all current articles, reviews and (text)books. The real physics should be what after taking the variational extreme values of physics systems, the physics systems' general extremum functional $F(G)$ needs to further take the general extremum functional $F$ 's ( $G$ 's ) minimum absolute extremum zero, otherwise, the appearing processes of physics systems still are virtual processes because the virtual process situations cannot deduce canonical equations and their corresponding conservation quantities. These conclusions for finite (infinite) freedom systems are not only the supplementary developments for the current canonical variational principle and current canonical Noether theorem, but also classsical and quantum new physics corresponding to classical and quantum physics systems in phase space.

Up to now, this paper uncovers that all the investigations about canonical variational principle and canonical Noether theorem for different physics systems with (in)finite freedom systems have missed the key investigations about doublet extremum processes of the general extreme value functional $F(G)$ that both is derived by the least canonical action principle and is necessarily largely taken in deriving all the physics laws in phase space, but which have no been done, which lead to the crisis of deriving all physics laws in phase space. Utilizing the studies above on the general extremum functional F's ( $G$ 's ) doublet extremum processes in this paper, namely, on the double extremums, the hidden logic cycle and the crisis are solved, and the new mathematical and physical pictures for (in)finite freedom systems are discovered. Therefore, for (in)finite freedom systems, the general canonical variantional principle and the general canonical Noether theorem are shown in phase space, which solve the hidden logic cycle and the crisis having existed for over a century since Noether's proposed her famous theorem.

This paper discovers the new equations of conservation currents of general canonical variational principle and general canonical Noether theorem and their new corresponding convervation currents, their conservation charges deduced from new conservation currents are the same as the conservation charges of the usual canonical variational principle and the usual canonical Noether theorem, which show that our studies are consistent with current canonical variational principle and current canonical Noether theorem.

Consequently, this paper opens a door of studying general canonical variational principle and general canonical Noether theorem of (in)finite freedom systems via taking the double extremum to show origins of physics laws in phase space, especially, all quantum physics laws need to be in phase space, and will rewrite significantly the relevant investigations of different sciences, because canonical variational principle and canonical Noether theorem for (in)finite freedom systems have been the key solid bases in physics or general sciences, and this paper just uncovers new avenues of investigations for (in)finite freedom systems, general canonical variational principle, general canonical Noether theorem and their applications in different sciences, and all the current relevant papers and (text)books need to rewrite, supply and update. 
Acknowledgments: The work is supported by NSF through grants PHY-0805948, DOE through grant DEFG02- 91ER40681 and National Natural Science Foun- dation of China (No. 11875081).
[1] Badin, Gualtiero; Crisciani, Fulvio, Variational Formulation of Fluid and Geophysical Fluid Dynamics - Mechanics, Symmetries and Conservation Laws, Springer, (2018)

[2] Morris Kline, Mathematical Thought from Ancient to Modern Times. New York: Oxford University Press (1972).

[3] Philippe Blanchard, G. M. Hayes, Variational Methods in Mathematical Physics: A Unified Approach (Theoretical and Mathematical Physics), Springer (2011).

[4] Richard Feynman, The Character of Physical Law, Modern Library, (1965), ISBN 0-679-60127-9.

[5] Noether, E., Invariante Variationsprobleme, Nachrichten von der Gesellschaft der Wissenschaften zu Gottingen, Mathematisch-Physikalische Klasse. 1918: 235-257.

[6] EDITORIAL, Celebrate the mathematics of Emmy Noether, Nature 561, 149-150 (2018), doi: 10.1038/d41586-018-06658-w.

[7] Emmy Noether; Mort Tavel (translator) (1971). "Invariant Variation Problems". Transport Theory and Statistical Physics. 1 (3): 186-207. arXiv:physics/0503066.

[8] Kosmann-Schwarzbach, Yvette. The Noether theorems: Invariance and conservation laws in the twentieth century. Sources and Studies in the History of Mathematics and Physical Sciences. Springer-Verlag, (2010).

[9] Ekeland, Ivar (1979). "Nonconvex minimization problems". Bulletin of the American Mathematical Society. New Series. 1 (3): 443-474.

[10] Yong-Chang Huang, Unified Expressions of All Differential Variational Principles, Mechanics Research Communications, 30, 6 (2003) 567, and its references.

[11] Yong-Chang Huang, X. G. Lee and M. X. Shao, Unified Expressions Of All Integral Variational Principles, Modern Physics Letters, A21(2006) 1107.

[12] Kevin W. Cassel, Variational Methods with Applications in Science and Engineering, Cambridge University Press, 2013.

[13] Sardanashvily, G. Noether's Theorems, Applications in Mechanics and Field Theory, Springer-Verlag, (2016).

[14] Hanca, J.; Tulejab, S.; Hancova, M., Symmetries and conservation laws: Consequences of Noether's theorem, American Journal of Physics. 72 (4): 428-35, (2004).

[15] Cornelius Lanczos, The Variational Principles of Mechanics (4th ed.). Dover. University of Toronto Press (1974).

[16] Adhikari S. K., Variational Principles for the Numerical Solution of Scattering Problems, New York: Wiley, 1998.

[17] Yong-Chang Huang, L. Liao and X. G. Lee, FaddeevJackiw canonical path integral quantization for a general scenario, its proper vertices and generating functionals, The European Physical Journal, C60 (2009) 481-487.

[18] Hand L. N., Finch J. D., Analytical Mechanics, England: Cambridge University Press, 2008.
[19] M. E. Peskin and D. V. Schroeder, An Introduction to Quantum Field Theory (Beijing World Publishing Corp., 2006).

[20] John Venables, "The Variational Principle and some applications". Dept of Physics and Astronomy, Arizona State University, Tempe, Arizona (Graduate Course: Quantum Physics)

[21] Nesbet R. K., Variational Principles and Methods In Theoretical Physics and Chemistry, New York: Cambridge U.P., 2003.

[22] Epstein S. T., The Variation Method in Quantum Chemistry". (New York: Academic, 1974).

[23] Marco Piccardo, Paul Chevalier, Benedikt Schwarz et al, Frequency-Modulated Combs Obey a Variational Principle, Phys. Rev. Lett. 122, 253901 (2019).

[24] Alexander G. Abanov and Gustavo M. Monteiro, FreeSurface Variational Principle for an Incompressible Fluid with Odd Viscosity, Phys. Rev. Lett. 122, 154501 (2019).

[25] Pablo M. Piaggi and Michele Parrinello, MultithermalMultibaric Molecular Simulations from a Variational Principle, Phys. Rev. Lett. 122, 050601 (2019).

[26] Dallas R. Trinkle, Variational Principle for Mass Transport, Phys. Rev. Lett. 121, 235901 (2018).

[27] Hendrik Weimer, Variational Principle for Steady States of Dissipative Quantum Many-Body Systems, Phys. Rev. Lett. 114, 040402 (2015).

[28] Jutho Haegeman, J. Ignacio Cirac, Tobias J. Osborne et al, Time-Dependent Variational Principle for Quantum Lattices, Phys. Rev. Lett. 107, 070601 (2011).

[29] Nvsen Ma, Yi-Zhuang You, and Zi Yang Meng, Role of Noether's Theorem at the Deconfined Quantum Critical Point, Phys. Rev. Lett. 122, 175701 (2019).

[30] Gáor Zsolt Tóh, Noether's theorems and conserved currents in gauge theories in the presence of fixed fields, Phys. Rev. D 96, 025018 (2017).

[31] C. Rudowicz and J. Qin, Noether's theorem and conserved quantities for the crystal- and ligand-field Hamiltonians invariant under continuous rotational symmetry, Phys. Rev. B 67, 174420 (2003).

[32] Kibble T. W. B., Classical Mechanics, European Physics Series, McGraw-Hill (UK), 1973, ISBN 0-07-084018-0.

[33] Gitman D. M., Tyutin I. V., Quantization of Fields with Constraints, Springer-Verlag, Berlin, 1990.

[34] Li Ziping and Jiang Jinhuan, Symmetries in Constrained Canonical Systems, Science Press, New York, 2002.

[35] C. Huang and Yong-Chang Huang, General variational principle, general Noether theorem, their classical and quantum new physics and solution to crisis deducing all physics laws, Preprints 2020, 2020080334 (doi: $\quad 10.20944 /$ preprints202008.0334.v3; https://www.preprints.org/manuscript/202008.0334/v3). 